\title{
Detailed state model of CaMKII activation and autophosphorylation
}

\author{
Vladan Lučić · Gabriela J. Greif · Mary B. Kennedy
}

Received: 14 December 2007/Revised: 2 July 2008/Accepted: 3 July 2008 / Published online: 4 September 2008

(C) The Author(s) 2008. This article is published with open access at Springerlink.com

\begin{abstract}
By combining biochemical experiments with computer modelling of biochemical reactions we elucidated some of the currently unresolved aspects of calciumcalmodulin-dependent protein kinase II (CaMKII) activation and autophosphorylation that might be relevant for its physiological function and provided a model that incorporates in detail the mechanism of CaMKII activation and autophosphorylation at T286 that is based on experimentally determined binding constants and phosphorylation rates. To this end, we developed a detailed state model of CaMKII activation and autophosphorylation based on the currently available literature, and constrained it with data from CaMKII autophosphorylation essays. Our model takes exact phosphorylation patterns of CaMKII holoenzymes into account, and is valid at physiologically relevant conditions where the concentrations of calcium and calmodulin are not saturating. Our results strongly suggest
\end{abstract}

Electronic supplementary material The online version of this article (doi:10.1007/s00249-008-0362-4) contains supplementary material, which is available to authorized users.

\section{Lučić · G. J. Greif · M. B. Kennedy}

Division of Biology, California Institute of Technology,

Pasadena, CA 91125, USA

Present Address:

V. Lučić (ه)

Department of Structural biology,

Max Planck Institute of Biochemistry,

Am Klopferspitz 18, 82152 Martinsried, Germany

e-mail: vladan@biochem.mpg.de

Present Address:

G. J. Greif

European Patent Office, Bayerstr. 34,

80335 Munich, Germany that even when bound to less than fully calcium-bound calmodulin, CaMKII is in the active state, and indicate that the autophosphorylation of T286 by an active non-phosphorylated CaMKII subunit is significantly faster than by an autophosphorylated CaMKII subunit. These results imply that CaMKII can be efficiently activated at significantly lower calcium concentrations than previously thought, which may explain how CaMKII gets activated at calcium concentrations existing at synapses in vivo. We also investigated the significance of CaMKII holoenzyme structure on CaMKII autophosphorylation and obtained estimates of previously unknown binding constants.

Keywords CaMKII $\cdot$ Calcium-calmodulindependent protein kinase - Computer simulation . Calcium signalling; modelling $\cdot$ Synapse

\section{Introduction}

$\mathrm{Ca}^{2+}$-calmodulin-dependent protein kinase II (CaMKII) is a multifunctional serine/threonine kinase. It is one of the key molecules involved in synaptic plasticity (Silva et al. 1992; Stevens et al. 1994; Hayashi et al. 2000), a process believed to be responsible for many forms of learning and memory. CaMKII is a major downstream effector of $\mathrm{Ca}^{2+}$ signaling and exerts its influence by phosphorylating several molecules present at neuronal synapses. Understanding the activation of CaMKII is therefore crucial for the understanding of the biochemical signaling pathways involved in synaptic plasticity.

CaMKII isolated from forebrains forms holoenzymes consisting of two major isoforms, $\alpha$ and $\beta$, present in a ratio of 3:1 (Bennett et al. 1983). In a single-particle cryo electron microscopy (EM) study, the number of subunits in 
a holoenzyme was estimated to be 12 , organized in a double ring with 6 subunits each (Kolodziej et al. 2000), consistent with earlier biochemical results (Bennett et al. 1983). More recently, the crystal structure of a holoenzyme formed by bacterially expressed $\alpha$ CaMKII association domains showed that the holoenzyme consists of 14 subunits organized in two closely apposed 7-subunit rings (Hoelz et al. 2003). However, another single particle EM study of negatively stained $\alpha$ - and $\beta$ CaMKII, offering higher contrast, strongly suggests that CaMKII holoenzymes are dodecamers (Gaertner et al. 2004a).

The main steps of CaMKII activation and autophosphorylation are well known. CaMKII is activated upon $\mathrm{Ca}^{2+}$ /calmodulin (CaM) binding. CaM can bind up to four $\mathrm{Ca}^{2+}$ ions with its four EF-hands. A CaMKII subunit remains active after it is autophosphorylated (at T286 on the $\alpha$-subunit, or T287 on $\beta$, both denoted T286 autophosphorylation) (Miller and Kennedy 1986; Lou et al. 1986). We will use the terms "active non-phospho" and "T286 phosphorylated" to distinguish between the active non-phosphorylated and active T286 phosphorylated forms of CaMKII. The T286 autophosphorylation is an intersubunit but intra-holoenzyme event, and it is believed to occur between neighboring subunits (Miller and Kennedy 1986; Rich and Schulman 1998; Bradshaw et al. 2002). In order to be phosphorylated at T286 a subunit has to be in the active non-phospho state, while the phosphorylating unit could be either an active non-phospho or an T286 phosphorylated subunit (Rich and Schulman 1998). Another physiologically relevant phosphorylation site is T305/306 at $\alpha$ CaMKII (T306/307 at $\beta$ CaMKII, both denoted as T305 phosphorylation) (Hashimoto et al. 1987; Lou and Schulman 1989). This is an intra-subunit phosphorylation that reduces the catalytic activity of CaMKII (Hashimoto et al. 1987; Lou and Schulman 1989). It can occur after CaM dissociates from a T286 autophosphorylated subunit, and it prevents the rebinding of $\mathrm{CaM}$ (Hashimoto et al. 1987; Patton et al. 1990).

Several models were developed for investigating various aspects of CaMKII function, such as the frequency response of CaMKII to $\mathrm{Ca}^{2+}$ oscillations (Hanson et al. 1994; Dosemeci and Albers 1996; Okamoto and Ichikawa 2000; Holmes 2000; Zhabotinsky 2000; Kubota and Bower 2001; Dupont et al. 2003; Miller et al. 2005), and the switch-like behavior of CaMKII in the presence of protein phosphatases (Okamoto and Ichikawa 2000; Holmes 2000; Zhabotinsky 2000; Kubota and Bower 2001; Miller et al. 2005). These models generally account for the intersubunit nature of CaMKII autophosphorylation at T286, and one of these models implements $\mathrm{Ca}-\mathrm{CaM}-\mathrm{CaMKII}$ binding in detail (Holmes 2000). However, these models are neither based on detailed phosphorylation patterns of CaMKII holoenzymes that include all possible arrangements of phosphorylated and non-phosphorylated CaMKII subunits, nor they consider T305 phosphorylated subunits (except Dosemeci and Albers 1996) or $\beta$ subunits. Furthermore, it would be of interest to explore aspects of CaMKII activation and autophosphorylation that are currently unresolved in the experimental literature. Therefore, there is a need for a detailed model of CaMKII activation and autophosphorylation valid under physiological conditions.

In this study, we combined biochemical experiments with computer modeling of biochemical reactions to achieve two goals. The first was to elucidate some of the currently unresolved aspects of CaMKII activation and autophosphorylation that are relevant for its physiological function, such as the activation of CaMKII in non-saturating $\mathrm{Ca}^{2+}$ conditions that are present in vivo, and the difference in phosphorylation rates between active nonphospho and T286 phosphorylated subunits. The second goal was to develop a model that provides a detailed account of the mechanism of CaMKII activation and T286 autophosphorylation and is based on experimentally determined binding constants and phosphorylation rates. In order to achieve these goals, we developed a detailed state model of CaMKII activation and autophosphorylation based on the currently available literature, and constrained it with data from our CaMKII autophosphorylation assays under different experimental conditions. This model takes exact phosphorylation patterns of CaMKII holoenzymes into account, and implements detailed $\mathrm{Ca}-\mathrm{CaM}-\mathrm{CaMKII}$ binding. It includes $\beta$ subunits and autophosphorylation at T305. Consequently, the model is valid at physiologically relevant conditions in which the concentrations of $\mathrm{Ca}^{2+}$ and $\mathrm{CaM}$ are not saturating.

\section{Materials and methods}

\section{CaMKII autophosphorylation assay}

Rat forebrain CaMKII was purified as described in (Bennett et al. 1983) with minor modifications (Miller and Kennedy 1985). Pure CaMKII was assayed at 25 and $30^{\circ} \mathrm{C}$ in a mixture (final volume $200 \mu \mathrm{l}$ ) containing $0.27 \mu \mathrm{M}$ CaMKII (subunit concentration), $1.2 \mu \mathrm{M}$ CaM (Calbiochem 208694), variable amounts of $\mathrm{CaCl}_{2}(0-0.7 \mathrm{mM})$, $1 \mathrm{mM} \mathrm{MgCl} 2,150 \mathrm{mM} \mathrm{KCl}, 0.4 \mathrm{mM}$ EGTA, $0.1 \mathrm{mM}$ ATP, $50 \mathrm{mM}$ Tris- $\mathrm{HCl}$ (pH 7.1), $0.6 \mathrm{mg} / \mathrm{ml} \mathrm{BSA}$ and $0.4 \mathrm{mM}$ DTT. All of the above ingredients except for CaMKII were mixed and prewarmed at the desired temperature (25 or $30^{\circ} \mathrm{C}$ ) for $2 \mathrm{~min}$. The reactions were initiated by the addition of CaMKII. The reactions were terminated by adding $3 \mathrm{x}$ stop solution (9\% SDS and $3 \% \mathrm{BME}$ ) at $30 \mathrm{~s}, 1$, 2 and $3 \mathrm{~min}$. The samples obtained this way are called reaction samples. The standard samples were prepared the 
same way except that when anti-non-phospho T286 antibodies were used for detection, the reactions contained no $\mathrm{CaCl}_{2}$, and they were stopped immediately after adding CaMKII. For phospho-T286 antibodies, detection standards contained $0.7 \mathrm{mM} \mathrm{CaCl} 2$ and the reaction time was 3 min. In both cases three standard samples were prepared, containing 100, 60 and $30 \%$ of the concentration of CaMKII present in the reaction samples. The viability of CaMKII during at least $3 \mathrm{~min}$ reaction time is supported by the following. CaMKII autophosphorylation reactions have been routinely carried at $30^{\circ} \mathrm{C}$ at longer times than what we used in several different groups without indications of protein structure breakdown (Miller and Kennedy 1986; Hashimoto et al. 1987; Lou and Schulman 1989; Meyer et al. 1992). In two of these studies, increased autophosphorylation was induced at 1 and 2 min after the reactions were started (Hashimoto et al. 1987; Lou and Schulman 1989). In addition, the reaction time recommended for commercially available CaMKII was $10 \mathrm{~min}$ (Upstate/ Millipore, product number 14-217).

\section{Quantitative Western blots}

The amount of T286 phosphorylated $\alpha$ CaMKII was determined by Western blots. Stopped samples were boiled for $2 \mathrm{~min}$ and centrifuged at 3,000 rpm for $1 \mathrm{~min}$. Equal volumes $(12 \mu \mathrm{l})$ of stopped (both reaction and standard) samples were loaded on a gel. Each gel contained three standard and 12 reaction samples. In this way, all reaction lanes contained the same amounts of $\alpha \mathrm{CaMKII}$ (1.6 pmol) and the three standard lanes contained 100, 60 and $30 \%$ of the $\alpha \mathrm{CaMKII}$ amount present in the reaction lanes. Samples were separated by SDS-PAGE under reducing conditions using $8 \%$ resolving gel, and electrophoretically transferred to PVDF membranes. The membranes were blocked for at least $2 \mathrm{~h}$ in 5\% nonfat milk in TTBS (20 mM Tris, $\mathrm{pH} 7.5$, $500 \mathrm{mM} \mathrm{NaCl}$ and $0.05 \%$ Tween 20) and incubated with appropriate primary antibodies. The primary antibodies used were rabbit anti non-phospho T286 $\alpha$ CaMKII (Darlene, 1:100-1: 200) and mouse anti phospho T286 CaMKII (clone 22B1, ABR MA1-047, 1:200) (Patton et al. 1993). Bound antibodies were detected by Alexa Fluor 488 (goat) conjugated anti-rabbit IgG (Molecular Probes A-11008, diluted with TTBS to $5 \mu \mathrm{g} / \mathrm{ml}$ ) and Alexa Fluor 488 (goat) conjugated anti-mouse IgG (Molecular Probes A-11001, diluted with TTBS to $5 \mu \mathrm{g} / \mathrm{ml}$ ) secondary antibodies and scanned on a Molecular Dynamics Storm system.

Image analysis was done using TNImage software (http:// brneurosci.org/tnimage.html). For each band, the (net) signal was calculated by subtracting the background value from the total signal of the $\alpha$ CaMKII band. A background value for a given band was obtained from a nearby area not containing any band, and adjusted to the area of the band.
The signals were corrected for the non-linearity of the scanner, according to the procedure implemented by the manufacturer.

For every anti non-phosphorylated T286 $\alpha$ CaMKII blot, a calibration curve was obtained from the best fit line for the signal obtained for the standard bands (corr. coef. $>0.99$ ). Figure $1 \mathrm{~b}$ shows that even a below-average fit shows linear behavior. The best fit line was used as a calibration allowing for the determination of the amount of non-phospho (and consequently phospho) T286 $\alpha$ CaMKII for all reaction bands present on the same blot (Table S3).

The best fit lines obtained from standards for each antiphospho T286 blot also showed linear behavior. The ratios of the lower and higher-mobility bands on anti-phospho T286 Western blots Fig. 1 were used to determine the amount of T305 phosphorylated $\alpha$ CaMKII. The lower mobility bands showed low signal at 0.5 and 1 min reaction times, so we only used the results at 2 and $3 \mathrm{~min}$.

\section{HPLC}

The autophosphorylation reactions were carried out essentially as above. The CaMKII autophosphorylation assay was modified in order to get a higher quantity of T286 phosphorylated CaMKII and to have roughly the same amounts of CaMKII in the both the lower and higher mobility $\alpha \mathrm{CaMKII}$ bands. The reaction mixtures contained 0.42-0.5 mM $\mathrm{CaCl}_{2}, \quad 6-12 \mu \mathrm{M} \quad \mathrm{CaM}, \quad 0.27-0.54 \mu \mathrm{M}$ CaMKII, $1 \mathrm{mM} \quad \mathrm{MgCl}_{2}, \quad 0.4 \mathrm{mM} \quad$ EGTA, $0.1 \mathrm{mM}$ $\left[\gamma-{ }^{32} \mathrm{P}\right]$ ATP $(1,500-3,000 \mathrm{cpm} / \mathrm{pmol}), 50 \mathrm{mM}$ Tris- $\mathrm{HCl}$ (pH 7.1), $0.6 \mathrm{mg} / \mathrm{ml} \mathrm{BSA}$ and $0.4 \mathrm{mM}$ DTT. The reactions were carried out at $25^{\circ} \mathrm{C}$ for $3 \mathrm{~min}$ or at $30^{\circ} \mathrm{C}$ for $1 \mathrm{~min}$.

After electrophoresis, the gels were stained in Coomassie and the bands corresponding to higher and lower mobility $\alpha \mathrm{CaMKII}$, as well as a control band containing no protein, were cut out and diced. The gel pieces were reduced and destained by reacting them in equal volumes of $4 \mathrm{mM}$ tris-(2-carboxyethyl) phosphine hydrochloride (TCEP) at $37^{\circ} \mathrm{C}$ for $40 \mathrm{~min}(\mathrm{pH}$ adjusted to $>8$ by $1 \mathrm{M}$ ammonium bicarbonate (AMBI)) and acetonitrile. The gel pieces were alkylated and further destained by reacting in $25 \mathrm{mM}$ AMBI and $25 \mathrm{mM}$ iodoacetamide (IAA) and 50\% acetonitrile for $35 \mathrm{~min}$ in the dark at room temperature and then rinsed a few times with $50 \mathrm{mM}$ AMBI. The gel pieces were lypophilized in SpeedVac for 4 h. $5 \mu$ l 0.2 M AMBI, $0.02 \%$ tween-20 was added to each tube containing dried gel pieces, immediately followed by the addition of $0.5-$ $1.5 \mu \mathrm{g}$ modified trypsin (20-fold excess of trypsin by weight) and 0.2 M AMBI was added several times during one hour course, until the samples were fully rehydrated. The digestion was carried out overnight at $30^{\circ} \mathrm{C}$. The reaction was stopped by the addition of $1 / 10$ vol of $10 \%$ TFA. The samples were spun at 1,500 rpm for $1 \mathrm{~min}$ and 
a

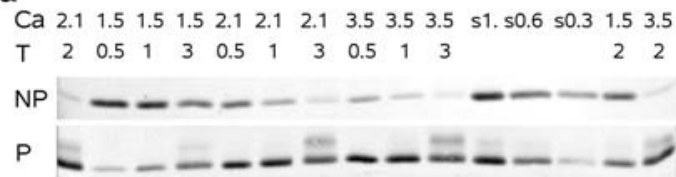

b
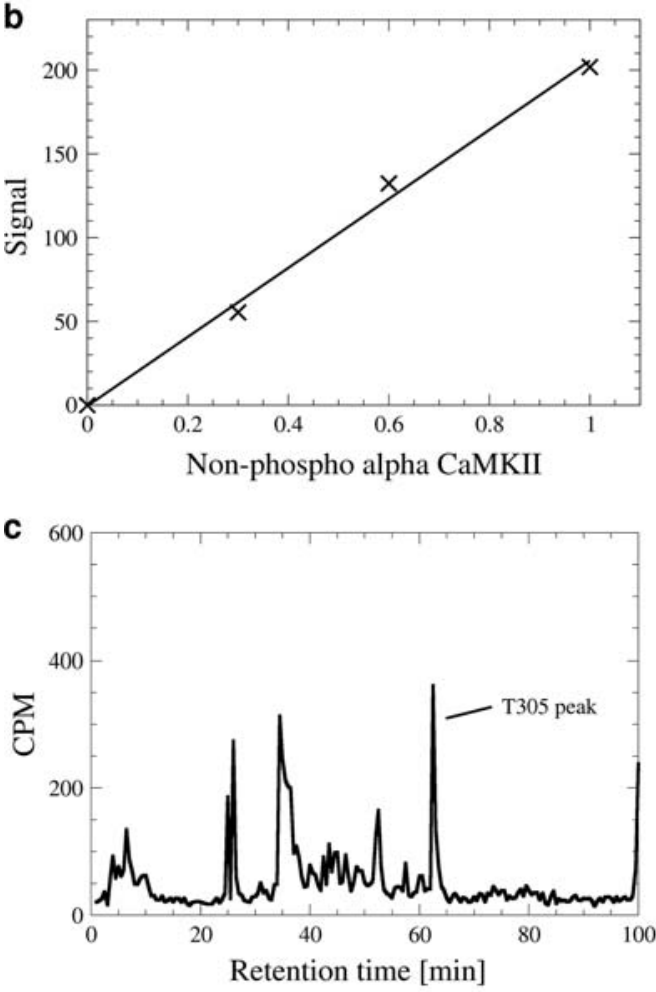

d

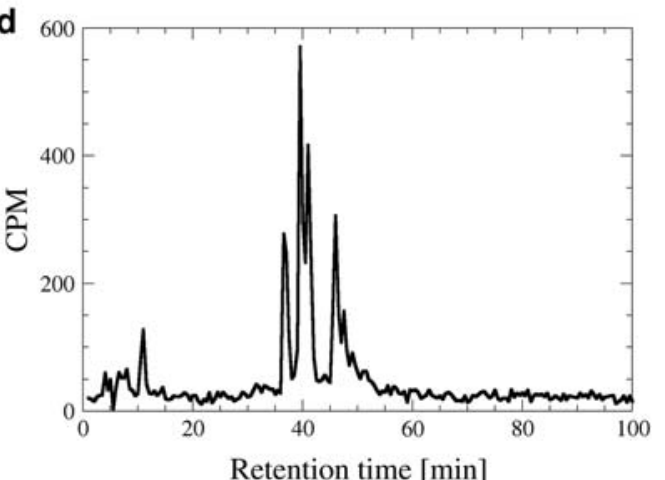

Fig. 1 Western blots and HPLC. a A representative anti non-phospho T286 (NP row) and phospho T286 $\alpha$ CaMKII Western blots (P row) containing samples obtained by CaMKII autophosphorylation assays for different free $\left[\mathrm{Ca}^{2+}\right]$ and reaction times at $25^{\circ} \mathrm{C}$ are shown. Lower and higher mobility bands can be seen in the anti phospho blot. Free $\left[\mathrm{Ca}^{2+}\right]$ in $\mu \mathrm{M}$ (top row) and the reaction times in min (second row) are shown for all reaction lanes. Lanes s1., s0.6 and s0.3 are standards containing 100, 60 and 30 of the CaMKII amount present in the other lanes (see "Materials and methods"). b A representative anti nonphospho T286 $\alpha$ CaMKII Western blot calibration curve was obtained from standard bands. The amount of non-phospho T286 $\alpha$ CaMKII ( $x$-axis) was normalized to 1 . $\mathbf{c}$ and $\mathbf{d}$ HPLC chromatography profiles of peptides from two anti phospho T286 $\alpha$ CaMKII bands showing the amount of incorporated ${ }^{32} \mathrm{P}$. The lower mobility band (c) shows T305 peak, but not the higher mobility band (d) the supernatant was saved. The remaining gel pieces were treated with $0.1 \%$ TFA and $60 \%$ acetonitrile at $30^{\circ} \mathrm{C}$ for $1 \mathrm{~h}$ and the supernatant was saved. Once again the remaining gel pieces were extracted with $75 \%$ acetonitrile at $30^{\circ} \mathrm{C}$ for $1 \mathrm{~h}$ and all the supernatants were combined. $1 \mathrm{M}$ DTT was added to the final concentration of few $\mu \mathrm{M}$. The organic phase was reduced by speed vacuum and the samples were filtered in Ultrafree-MC Milipore $(0.22 \mu \mathrm{m}$ filter) for $5 \mathrm{~min}$ at $12,000 \mathrm{rpm}$.

Reverse phase HPLC fractionation was carried out essentially as in Patton et al. (1990). Samples were loaded onto a $\mathrm{C} 4$ reverse-phase HPLC column equilibrated in buffer $\mathrm{A}\left(0.07 \% \mathrm{v} / \mathrm{v}\right.$ TFA in HPLC grade $\left.\mathrm{H}_{2} \mathrm{O}\right)$. Buffer $\mathrm{B}$ consisted of $0.07 \% \mathrm{v} / \mathrm{v}$ TFA, $70 \%$ acetonitrile, $30 \%$ HPLC grade $\mathrm{H}_{2} \mathrm{O}$. The column was developed at a flow rate of $1 \mathrm{ml} / \mathrm{min}$ with the following gradient: $0-18 \mathrm{~min} 5 \%$ buffer B, 18-95 min 5-35\% buffer B, 95-100 min 35-100\% buffer B. Labeled peptides were detected in the fractions by Cerenkov radiation.

\section{Computer simulations}

The models were implemented in the Mathematica environment on a Linux workstation (Intel based dual $450 \mathrm{MHz}$ processors). The differential and algebraic equations were solved numerically using Mathematica built-in functions. In particular, the differential equations for the CaMKII autophosphorylation model and for the complete model were solved using the Adams and Gear method. The equilibrium Ca-CaM-CaMKII model was analytically reduced to a nonlinear algebraic equation and solved using a damped Newton's, the secant and the Brent's methods. The individual simulations took up to a few seconds of computational time. The software is available upon request.

Determination of free $\mathrm{Ca}^{2+}$

Affinities of EGTA for $\mathrm{Ca}^{2+}$ and $\mathrm{Mg}^{2+}$ at $\mathrm{pH} 7.1$ were calculated using the affinities of EGTA for $\mathrm{Ca}^{2+}$ and $\mathrm{Mg}^{2+}$ (without $\mathrm{H}^{+}$), and for $\mathrm{H}^{+}$(for ionic strength of $0.1 \mathrm{M}$ and a temperature of $25^{\circ} \mathrm{C}$ ) given in (Martell and Smith 1982). For $30^{\circ} \mathrm{C}$, first the EGTA affinities for $\mathrm{H}^{+}, \mathrm{Ca}^{2+}$, and $\mathrm{Mg}^{2+}$ (without $\mathrm{H}^{+}$) were calculated using the values for $25^{\circ} \mathrm{C}$ and the enthalpy changes, and then the EGTA affinities for $\mathrm{Ca}^{2+}$ and $\mathrm{Mg}^{2+}$ at $\mathrm{pH} 7.1$ were calculated. The results are shown in Table S1. Binding of the third and forth proton to EGTA did not change the calculated affinities. Binding of $\mathrm{Ca}^{2+}$ to EGTA was reported to occur at a millisecond time scale for sub-millimolar EGTA and $\mathrm{Ca}^{2+}$ concentrations (Smith et al. 1984) and is therefore much faster than the autophosphorylation of CaMKII under our experimental conditions.

Free $\mathrm{Ca}^{2+}$ in the buffer used for the CaMKII autophosphorylation assay, with or without CaM and CaMKII, 
was calculated using mass-action equations for the simultaneous binding of $\mathrm{Ca}^{2+}$ and $\mathrm{Mg}^{2+}$ to EGTA and $\mathrm{Ca}^{2+}$ to $\mathrm{CaM}$ and CaM-CaMKII, that is without assuming that the $\mathrm{Ca}^{2+}$ buffering by EGTA was ideal. The concentrations of the reagents were identical to those present in the autophosphorylation assays. The dissociation constants of 0-3 $\mathrm{Ca}^{2+}$ bound $\mathrm{CaM}$ for $\mathrm{Ca}^{2+}$, in the presence of $1 \mathrm{mM} \mathrm{Mg}^{2+}$ at $25^{\circ} \mathrm{C}$, are: $6,4.2,77$ and $14.3 \mu \mathrm{M}$, respectively, and the off rates are: 40, 40, 600 and 600/s (Klee 1988). Since the $\mathrm{Ca}^{2+}-\mathrm{CaM}$ binding constants at $30^{\circ} \mathrm{C}$ were not available we used the same values for both temperatures. Affinities of $\mathrm{Ca}^{2+}$ for $0-3 \mathrm{Ca}^{2+}$ bound CaM, bound to phospho CaMKII, were taken to be 10 times higher than those without CaMKII, resulting in a 10,000 fold affinity increase of autophosphorylated CaMKII for CaM upon full binding of $\mathrm{Ca}^{2+}$, consistent with the published data (Meyer et al. 1992; Singla et al. 2001). The equations were solved numerically for three different cases: $\mathrm{CaM}(-) / \mathrm{CaMKII}(-), \mathrm{CaM}(+) /$ CaMKII $(-)$, and $\mathrm{CaM}(+) / \mathrm{CaMKII}(+\mathrm{P} 286)(\mathrm{CaMKII} 100 \%$ autophosphorylated at T286), and the results are shown in Table S2.

$\mathrm{CaM}$ increases its affinity for $\mathrm{Ca}^{2+}$ when bound to CaMKII, so the $\mathrm{CaM}(+) / \mathrm{CaMKII}(-)$ case overestimates the free $\mathrm{Ca}^{2+}$ in our experiments. CaMKII autophosphorylated at T286 but not at T305 has even higher affinity for $\mathrm{Ca}^{2+}$ (Meyer et al. 1992). Because CaMKII is never 100\% autophosphorylated at T286 and 0\% phosphorylated at $\mathrm{T} 305$, the $\mathrm{CaM}(+) / \mathrm{CaMKII}(+\mathrm{P} 286)$ case underestimates free $\mathrm{Ca}^{2+}$. The free $\mathrm{Ca}^{2+}$ in each of our experiments might vary between the values calculated for the last two cases, but considering that the difference between these two calculated values is small, we can assume that free $\mathrm{Ca}^{2+}$ in our experiments is time-independent, and that it is given by an average of the two calculated values.

\section{Results}

CaMKII autophosphorylation measurements

CaMKII autophosphorylation assays were performed at a non-saturating $\mathrm{CaM}$ concentration $(1.2 \mu \mathrm{M})$, for $\mathrm{Ca}^{2+}$ concentrations between 0.9 and $4.6 \mu \mathrm{M}$, different reaction times (between $30 \mathrm{~s}$ and $3 \mathrm{~min}$ ), and at different temperatures $\left(25\right.$ and $30^{\circ} \mathrm{C}$ ). We used $\mathrm{Ca}^{2+}$ concentrations resulting in a gradual autophosphorylation during the time course of the experiments. They are in the same range as those in central nervous system (CNS) synapses $(50 \mathrm{nM}$ baseline, upon stimulation up to $10 \mu \mathrm{M}$ at both pre- and postsynaptic terminals (Bollmann et al. 2000; Schneggenburger and Neher 2000; Sabatini et al. 2002)).

The concentrations of $\alpha \mathrm{CaMKII}$ autophosphorylated at T286 under different experimental conditions and at different time points were obtained form quantitative Western blots with anti non-phospho T286 $\alpha$ CaMKII antibodies (Fig. 1a). A calibration curve relating the signal intensity to a non-phospho CaMKII amount of a band was obtained for each blot (see "Materials and methods"). The calibration curves showed linear behavior (see Fig. 1b), so we used them to calculate the concentrations of nonphospho (and consequently also T286 phosphorylated) $\alpha$ CaMKII (Table S3).

Western blots using anti-phospho T286 CaMKII antibodies showed two close $\alpha$ CaMKII bands (Fig. 1a). The appearance on the lower mobility band was correlated earlier with phosphorylation of sites other than T286, and with conditions that promote autophosphorylation at T305 (chelating $\mathrm{Ca}^{2+}$ during CaMKII autophosphorylation) (Bennett et al. 1983; Hashimoto et al. 1987; Lou and Schulman 1989). Using HPLC fractionation of the tryptic phosphopeptides from both bands we confirmed that the lower mobility band, but not the higher mobility band, contains $\alpha$ CaMKII phosphorylated at T305. In each of the three experiments, the HPLC fractions of the lower mobility band contained one or two peaks at positions 62 $64 \mathrm{~min}$ and 79-81 min (Fig. 1c), consistent with two previously identified positions of the T305 containing $\alpha$ CaMKII fragments (see Figure 1E and 4D in Patton et al. 1990). None of the higher mobility band profiles had peaks at any of these two positions (Fig. 1d), while both higher and lower mobility bands showed peaks at positions between 30 and $50 \mathrm{~min}$. These were previously shown to correspond to $\alpha$ CaMKII fragments containing T286 (see Figure 1E in Miller et al. 1988 and 1C in Patton et al. 1990).

The ratio between the signals obtained from the higher and lower mobility bands of the anti-phospho T286 CaMKII Western blots, together with the results from antinon-phospho CaMKII Western blots, allowed us to estimate the concentrations of $\alpha$ CaMKII autophosphorylated at T305, under our experimental conditions (Table S3).

It has been shown that the phosphorylation at T305 occurs at essentially zero $\mathrm{Ca}^{2+}$ concentration $(500 \mu \mathrm{M}$ total $\mathrm{Ca}^{2+}$ and 10 mM EGTA in Lou and Schulman 1989, similar conditions in Patton et al. 1990), and is very slow at saturating $\mathrm{Ca}^{2+}$ (phosphate incorporation was not detected by autoradiography after $4 \mathrm{~min}$ in Lou and Schulman 1989). However, what happens at intermediate $\mathrm{Ca}^{2+}$ concentrations has not been investigated systematically yet. The substantial autophosphorylation at T305 that we observe under our experimental conditions can be explained by the higher dissociation rate of $\mathrm{CaM}$ (in respect to the saturating $\mathrm{Ca}^{2+}$ conditions) and the consequent T305 phosphorylation. We cannot exclude the possibility that the exact amount of T305 phosphorylated CaMKII was determined inaccurately because the 
anti-phospho T286 CaMKII antibody has different affinity for T305 phosphorylated $\alpha$ CaMKII. However, this would not change the outcome of our simulations because, as we show below, that autophosphorylation at T305 does not significantly affect the autophosphorylation at T286 under our experimental conditions.

\section{Modeling strategy}

Activation and autophosphorylation of CaMKII was conceptually separated into two parts. The first was the binding of $\mathrm{Ca}^{2+}$ to $\mathrm{CaM}$ and of $\mathrm{CaM}$ to non-phospho CaMKII $(\mathrm{Ca}-$ CaM-CaMKII binding). The second concerned the autophosphorylations at T286 and T305. In principle, starting from the concentrations of reactants and the binding constants for $\mathrm{Ca}-\mathrm{CaM}-\mathrm{CaMKII}$ binding, the fraction of active non-phospho CaMKII subunits $r$ (defined precisely below) is calculated using the $\mathrm{Ca}-\mathrm{CaM}-\mathrm{CaMKII}$ binding model. The CaMKII phosphorylation model requires that the values for $r$ as well as the phosphorylation rates are known, in order to calculate the time-course of CaMKII autophosphorylation (Fig. 2, filled arrows).

Since not all of the $\mathrm{Ca}-\mathrm{CaM}-\mathrm{CaMKII}$ binding constants are known, our simulation strategy was the following: first we ran a series of simulations of the CaMKII autophosphorylation model, for different values of the fractions $r$. We determined the values of $r$ under different experimental conditions by fitting the simulation results to our experimental CaMKII autophosphorylation data (parameter search for $r$ ). Next, the simulations using the $\mathrm{Ca}-\mathrm{CaM}-$ CaMKII binding model for different values of the unknown binding constants were used to find $r$ 's that are consistent with the previously obtained values (parameter search for the unknown -CaM-CaMKII binding constants). This way

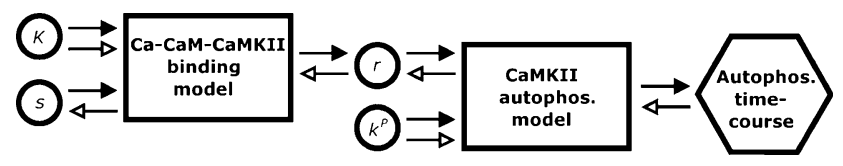

Fig. 2 Modeling strategy. Filled arrows work-flow for a case when all binding constants and phosphorylation rates are known. Starting with $\mathrm{Ca}-\mathrm{CaM}-\mathrm{CaMKII}$ binding constants $(K$ and $s)$, the $\mathrm{Ca}-\mathrm{CaM}-$ CaMKII binding model is used to calculate fractions $r$. These fractions, together with autophosphorylation rates $\left(k^{P}\right)$, are then used to obtain CaMKII autophosphorylation time-courses using the CaMKII autophosphorylation model. The only difference between this and the approach used for the simulations based on the complete dynamic model is that in the later case the $\mathrm{Ca}-\mathrm{CaM}-\mathrm{CaMKII}$ binding and the CaMKII autophosphorylation models are integrated in one, complete model. Empty arrows work-flow used to determine some of the binding constants. First the CaMKII autophosphorylation model is used to find the values of the fractions $r$ that are consistent with the experimentally obtained CaMKII autophosphorylation time-courses. Then, the $\mathrm{Ca}-\mathrm{CaM}-\mathrm{CaMKII}$ binding model is used to find the values of the previously undetermined $\mathrm{Ca}-\mathrm{CaM}-\mathrm{CaMKII}$ binding constants $(s)$, using known $\mathrm{Ca}-\mathrm{CaM}-\mathrm{CaMKII}$ binding constants $(K)$ we determined the values of the previously unknown binding constants (Fig. 2, empty arrows).

\section{Detailed state model of $\mathrm{Ca}-\mathrm{CaM}-\mathrm{CaMKII}$ binding}

The detailed state model of $\mathrm{Ca}-\mathrm{CaM}-\mathrm{CaMKII}$ binding presented here describes $\mathrm{Ca}^{2+}$ binding to $\mathrm{CaM}, \mathrm{CaM}$ (with $0-4$ bound $\mathrm{Ca}^{2+}$ ions) binding to both the $\alpha$ and $\beta$ isoforms of CaMKII, and $\mathrm{Ca}^{2+}$ binding to (0-3 $\mathrm{Ca}^{2+}$ bound) $\mathrm{CaM}-$ CaMKII (both isoforms).

According to earlier results, the binding of $\mathrm{Ca}^{2+}$ to $\mathrm{CaM}$ was taken to be ordered and cooperative (Klee 1988). Both low and high affinity $\mathrm{Ca}^{2+}$ binding sites are composed of two cooperative binding sites. Binding of CaMKII to CaM was modeled in a similar way to CaM binding to other enzymes, such as cAMP phosphodiesterase (PDE) and myosin light chain kinase (MLCK) (Wang et al. 1980; Gregori et al. 1985; Cox 1988). Upon $\mathrm{Ca}^{2+}$ binding, $\mathrm{CaM}$ adopts an open confirmation exposing a hydrophobic surface. Enzyme binding hides this surface and consequently increases the affinity of $\mathrm{CaM}$ for $\mathrm{Ca}^{2+}$. Likewise, $\mathrm{Ca}^{2+}$ binding increases the affinity of $\mathrm{CaM}$ for the enzyme (Lukas et al. 1998). Our Ca-CaM-CaMKII binding model was more complicated because binding of CaM to both $\alpha$ and $\beta$ isoforms of CaMKII was considered. This was necessary because $\beta$ CaMKII is known to have higher affinity for $\mathrm{CaM}$. The mass-action equations for $\mathrm{Ca}-\mathrm{CaM}-$ CaMKII (system of 15 nonlinear first-order differential equations) were obtained directly from the $\mathrm{Ca}-\mathrm{CaM}-$ CaMKII reaction scheme (Fig. 3). The concentrations of free $\mathrm{Ca}^{2+}$ and free CaMKII were not dynamic variables because the free $\mathrm{Ca}^{2+}$ concentration changed only slightly during the course of our experiments (see "Materials and methods"). In case where $\mathrm{Ca}^{2+}, \mathrm{CaM}$ and CaMKII are in equilibrium, the resulting system of the algebraic equations is called the equilibrium $\mathrm{Ca}-\mathrm{CaM}-\mathrm{CaMKII}$ model. It was implicitly assumed that the binding of $\mathrm{Ca}-\mathrm{CaM}$ to a CaMKII subunit did not depend on the phosphorylation states of other subunits, which is justified by the finding that the binding of CaM to CaMKII is non-cooperative (Bradshaw et al. 2003).

Affinities of $0-3$ bound $\mathrm{CaM}$ for $\mathrm{Ca}^{2+}\left(K_{01}-K_{34}\right)$ are given in Klee (1988) (see "Materials and methods"). $s_{1}-s_{4}$ are affinity increase factors that determine the affinity increase of the $0-3$ bound $\mathrm{CaM}$ for $\mathrm{Ca}^{2+}$ upon CaMKII binding. We assumed that they were the same for both isoforms. Therefore, the affinities of 0-3 bound CaMCaMKII for $\mathrm{Ca}^{2+}$ were $s_{1} K_{01}-s_{4} K_{34}$. Based on the increased affinity of $\mathrm{CaM}$ for $\mathrm{Ca}^{2+}$ upon $\mathrm{CaM}$ binding to MLCK (Mamar-Bachi and Cox 1987), and the homology of CaM binding domains of MLCK and CaMKII, CaMKII binding is expected to increase the affinity of $0-3$ bound $\mathrm{CaM}$ for $\mathrm{Ca}^{2+}$. For thermodynamic reasons, the affinities 


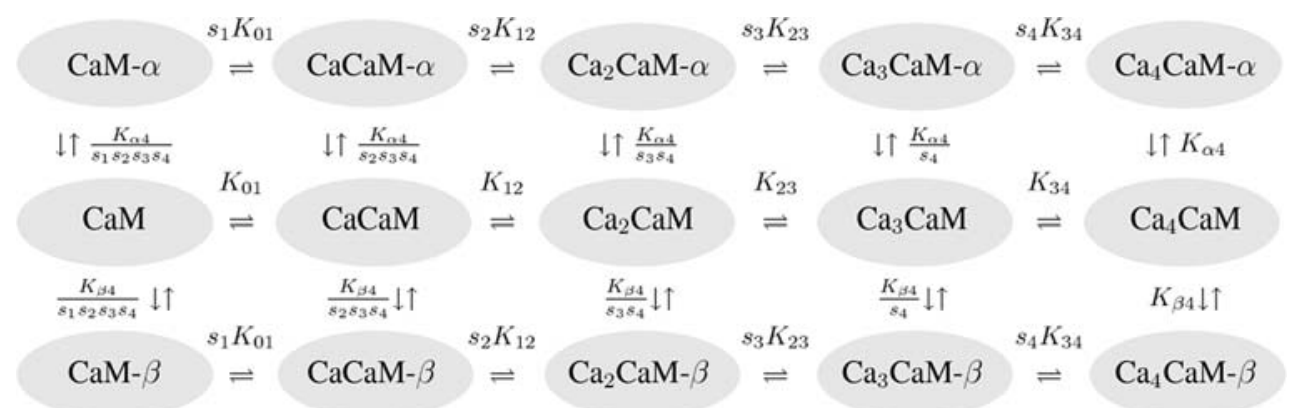

Fig. 3 The reaction scheme for $\mathrm{Ca}-\mathrm{CaM}-\mathrm{CaMKII}$ binding model. The complete scheme of $\mathrm{Ca}^{2+}$ binding to $\mathrm{CaM}, \mathrm{CaM}$ (with or without $\mathrm{Ca}^{2+}$ ) binding to both $\alpha$ and $\beta$ isoforms of CaMKII and $\mathrm{Ca}^{2+}$ binding to CaM-CaMKII (both isoforms) is shown. This scheme contains 15 states defined by the number of $\mathrm{Ca}^{2+}$ ions bound $(0-4)$ and the CaMKII binding (none, $\alpha$, or $\beta$ isoforms). In the scheme $\alpha$ and $\beta$ stand for $\alpha \mathrm{CaMKII}$ and $\beta \mathrm{CaMKII}$, respectively. Concentrations of free

of $\alpha$ - and $\beta$ CaMKII for $0-3$ bound CaM can be expressed in terms of the CaMKII affinity for 4-bound CaM $\left(K_{\alpha 4}\right.$ and $K_{\beta 4}$ ) and the affinity increase factors $s_{1}-s_{4}$ (Fig. 3).

In order to determine $K_{\alpha 4}$ and $K_{\beta 4}$, we performed numerical simulations of the experiments used to produce Fig. 3 in Meyer et al. (1992) using our Ca-CaM-CaMKII binding model. Quantitative agreement with the data was obtained for $1 / K_{\alpha, 4}=20 \mathrm{nM}$ (on-rate $150 \times 10^{6} / \mathrm{Ms}$ (Meyer et al. 1992)) and for the increase of affinity of CaMKII for CaM upon binding four $\mathrm{Ca}^{2+}$ ions (product of all affinity increase factors $s_{1} s_{2} s_{3} s_{4}$ ) between 16 and 1,000. These values might appear different from the values reported in Meyer et al. (1992): the dissociation constant of $43 \mathrm{nM}$ at the highest $\left[\mathrm{Ca}^{2+}\right]$ (adjusted for the fact that CaMKII has a 3-times lower affinity for wildtype $\mathrm{CaM}$ than for the dansylated $\mathrm{CaM}$ used in these experiments) and the threefold decrease in the dissociation rate between the lowest and the highest $\left[\mathrm{Ca}^{2+}\right]$. There are two reasons for this apparent discrepancy. First, the highest $\left[\mathrm{Ca}^{2+}\right]$ used in Meyer et al. (1992), $10 \mu \mathrm{M}$, is not saturating. Second, the dissociation rate measured in Meyer et al. (1992) is a combination of two dissociation rates: the $\mathrm{Ca}^{2+}$ dissociation from $\mathrm{CaM}$ and the $\mathrm{CaM}$ dissociation from CaMKII. Both rates are fast and have comparable values, so the values reported in (Meyer et al. 1992) are likely to overestimate the dissociation constant $1 / K_{\alpha 4}$ and underestimate the affinity change. In another publication $1 / K_{\alpha 4}$ was estimated to be $60 \mathrm{nM}$ (Gaertner et al. 2004a). We used both 20 and $60 \mathrm{nM}$ in our simulations. The affinity of $\beta \mathrm{CaMKII}$ for $\mathrm{CaM}$ was in both cases taken to be ten times higher than that of $\alpha$ CaMKII, consistent with the approximately ten times lower $\mathrm{EC}_{50}$ for $\beta$ CaMKII (Brocke et al. 1999). The exact values for the $\beta$ subunits did not influence our results much, since there were three times more $\alpha$ than $\beta$ subunits.
$\mathrm{Ca}^{2+}$ and CaMKII are not shown explicitly in the scheme, but it is understood that they are taken into account for $\mathrm{Ca}^{2+}$ and CaMKII bindings, respectively. The binding affinities are indicated next to the arrows: $K_{01}-K_{34}$ represent the affinity of $0-3$ bound $\mathrm{CaM}$ for $\mathrm{Ca}^{2+}$, $s_{1} K_{01}-s_{4} K_{34}$ are the affinities of $0-3$ bound CaM-CaMKII for $\mathrm{Ca}^{2+}$, and $K_{\alpha 4}\left(K_{\beta 4}\right)$ is $\alpha(\beta)$ CaMKII affinity for 4-bound CaM

\section{CaMKII autophosphorylation model}

The states of this model are distinguished by the arrangement of non-phosphorylated, T286 phosphorylated, and T305 phosphorelated subunits. They were obtained by splitting the states of a simplified model that did not take into account the T305 phosphorylation (Fig. 4a) according to the arrangement of the T305 phosphorylated states (as shown in Fig. 4b,c), and yielded a total of 38 states. We distinguished only those T305 phosphorylated subunits that were relevant for the T286 phosphorylation of another subunit, that is if a given subunit was next to a non-phosphorylated subunit (in the direction of the phosphorylation) so that it could phosphorylate another subunit.

Subunit autophosphorylations by active non-phospho, T286 and T305 phosphorylated subunits were represented as transitions between states. A transition weight was associated with each transition and it denoted the number of ways that the transition could occur (Fig. 4; Eqs. S2-S5).

Following the standard mass-action formalism, the rate of increase of the concentration of a state was given by the product of the initial states (38-dimensional vector $\Lambda$, normalized so that the sum of all elements equals 1 ), phosphorylation rates $(k ' s)$ and stoichiometric factors ( $Q$ 's), to yield:

$$
\begin{aligned}
\frac{\mathrm{d} \Lambda}{\mathrm{d} t}= & k_{\mathrm{aa}}^{P}\left(\frac{r_{\alpha} \alpha_{\mathrm{np}}+r_{\beta} \beta_{\mathrm{np}}}{\alpha_{\mathrm{np}}+\beta_{\mathrm{np}}}\right)^{2} Q_{\mathrm{aa}} \Lambda+k_{\mathrm{ap}}^{P} \frac{r_{\alpha} \alpha_{\mathrm{np}}+r_{\beta} \beta_{\mathrm{np}}}{\alpha_{\mathrm{np}}+\beta_{\mathrm{np}}} Q_{\mathrm{ap}} \Lambda \\
& +k_{\mathrm{app}}^{P} \frac{r_{\alpha} \alpha_{\mathrm{np}}+r_{\beta} \beta_{\mathrm{np}}}{\alpha_{\mathrm{np}}+\beta_{\mathrm{np}}} Q_{\mathrm{app}} \Lambda+k_{\mathrm{eff}}^{\mathrm{PP}} Q_{\mathrm{pp}} \Lambda
\end{aligned}
$$

T286 phosphorylation by active non-phospho subunits (the first term on the right hand side) is characterized by phosphorylation rate $k_{\mathrm{aa}}^{\mathrm{P}}$ and transition matrix $Q_{\mathrm{aa}}$. 


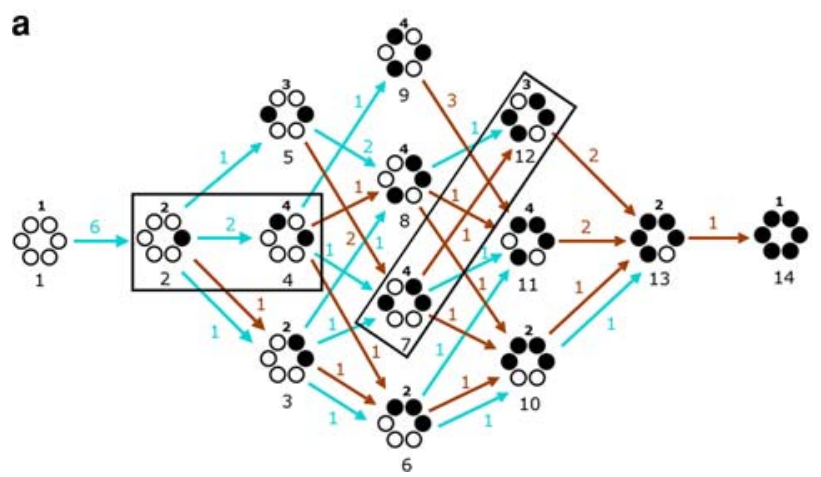

b

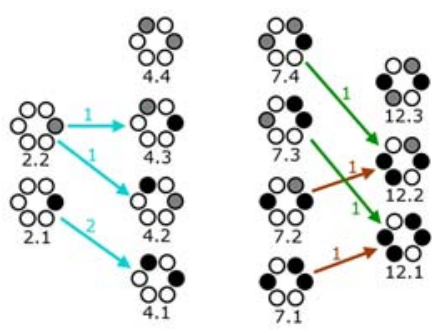

Fig. 4 CaMKII autophosphorylation scheme. The states of the CaMKII autophosphorylation model are distinguished by the number and positioning of non-phosphorylated (empty circles), T286 phosphorylated (black), and relevant T305 phosphorylated subunits (grey). The states are numbered by the numbers placed directly below them. Transitions between the states, corresponding to the phosphorylations at T286 by active non-phospho (blue arrows), T286 phosphorylated (brown arrows), and T305 phosphorylated (green arrows) CaMKII subunits, together with their weights, or number of ways transitions can occur (numbers next to the arrows) are also shown. The phosphorylation proceeds in the counter-clockwise direction. a All states and transitions of the simplified CaMKII autophosphorylation model (no T305 phosphorylation). The number of states that each state splits into when the autophosphorylation at T305 is taken into account is shown above the states. b and c States and transitions obtained upon the inclusion of the T305 autophosphorylation that correspond to the boxed states of $\mathbf{a}$

$$
\begin{aligned}
\frac{\mathrm{d}}{\mathrm{d} t}\left(\alpha_{t}-\alpha_{\mathrm{np}}\right) & =\frac{r_{\alpha} \alpha_{\mathrm{np}}}{r_{\alpha} \alpha_{\mathrm{np}}+r_{\beta} \beta_{\mathrm{np}}} \frac{\mathrm{d} \Lambda}{\mathrm{d} t} \cdot N_{p} \frac{\alpha_{t}+\beta_{t}}{6} \\
\frac{\mathrm{d}}{\mathrm{d} t}\left(\beta_{t}-\beta_{\mathrm{np}}\right) & =\frac{r_{\beta} \beta_{\mathrm{np}}}{r_{\alpha} \alpha_{\mathrm{np}}+r_{\beta} \beta_{\mathrm{np}}} \frac{d \Lambda}{\mathrm{d} t} \cdot N_{p} \frac{\alpha_{t}+\beta_{t}}{6}
\end{aligned}
$$

where $\alpha_{t}$ and $\beta_{t}$ are the total concentrations of the $\alpha \mathrm{CaMKII}$ and $\beta$ CaMKII isoforms and $N_{p}$ is the vector containing the number of phosphorylated subunits for each state. The right hand sides were formed by a product of the rate of change of the concentration of all T286 phosphorylated subunits $\left(\frac{\mathrm{d} \Lambda}{\mathrm{d} t} \cdot N_{p} \frac{\alpha_{t}+\beta_{t}}{6}\right)$, and the probabilities that a subunit phosphorylated at T286 is the $\alpha$ (upper equation) or $\beta$ (lower equation) isoform. We assumed that the arrangement of the CaMKII isoforms in a holoenzyme did not follow any special pattern since there is no experimental data that would suggest that.

The T305 autophosphorylation was represented by the following equations:

$$
\begin{aligned}
& \frac{\mathrm{d} \alpha_{\mathrm{pp}}}{\mathrm{d} t}=k_{\mathrm{eff}}^{\mathrm{PP}}\left(\alpha_{t}-\alpha_{\mathrm{np}}-\alpha_{\mathrm{pp}}\right) \\
& \frac{\mathrm{d} \beta_{\mathrm{pp}}}{\mathrm{d} t}=k_{\mathrm{eff}}^{\mathrm{PP}}\left(\beta_{t}-\beta_{\mathrm{np}}-\beta_{\mathrm{pp}}\right)
\end{aligned}
$$

where $\alpha_{\mathrm{pp}}$ and $\beta_{\mathrm{pp}}$ are the concentrations of T305 phosphorylated $\alpha \mathrm{CaMKII}$ and $\beta$ CaMKII, respectively.

We can exploit the symmetry present in Eq. 1 by defining the normalized parameters indicated by superscript ${ }^{(0)}$ :

$$
\begin{aligned}
k_{\mathrm{ap}}^{\mathrm{P}(0)}= & \frac{k_{\mathrm{ap}}^{\mathrm{P}}}{\left(k_{\mathrm{aa}}^{\mathrm{P}}\right)^{1 / 2}} \quad k_{\text {app }}^{\mathrm{P}(0)}=\frac{k_{\text {app }}^{\mathrm{P}}}{\left(k_{\mathrm{aa}}^{\mathrm{P}}\right)^{1 / 2}} \\
& r_{\alpha}^{(0)}=\left(k_{\mathrm{aa}}^{\mathrm{P}}\right)^{1 / 2} r_{\alpha} \quad r_{\beta}^{(0)}=\left(k_{\mathrm{aa}}^{\mathrm{P}}\right)^{1 / 2} r_{\beta}
\end{aligned}
$$
describe the time courses of the total T286 phosphorylation of the isoforms are needed: 
The substitution of the above definitions into the Eq. 1 led to the elimination of parameter $k_{\mathrm{aa}}^{\mathrm{P}}$. In other words, if the CaM affinity for CaMKII is increased and the T286 phosphorylation rate is decreased in an appropriate way (by the square of the affinity increase factor), the phosphorylation time course of CaMKII remains unchanged.

Equations 1-3 describe mathematically the phosphorylations at T286 and T305, taking into account the phosphorylation states of individual subunits and their arrangement within a holoenzyme, and include the contributions of both $\alpha$ and $\beta$ isoforms. The equations constitute a system of 42 first-order nonlinear differential equations with the same number of unknown time-dependent functions (38 elements of $\Lambda, \alpha_{\mathrm{np}}, \beta_{\mathrm{np}}, \alpha_{\mathrm{pp}}$ and $\beta_{\mathrm{pp}}$ ). The solution of this model gives the time courses of all individual states and therefore also the time course of the total phosphorylation.

Determination of CaMKII autophosphorylation model parameters

Here we first extract the values of some biochemical constants from the current literature, and then we proceed to determine the remaining parameters from our data.

In order to determine the T286 phosphorylation rate at $25^{\circ} \mathrm{C}$ (Table S5), we calculated enthalpy from the experimentally determined data for 23 and $30^{\circ} \mathrm{C}$ in Bradshaw et al. (2002) This experimental data was obtained under the implicit assumption that the T286 phosphorylation rates of both active non-phospho $\left(k_{\mathrm{aa}}^{\mathrm{P}}\right)$ and T286 phosphorylated subunits $\left(k_{\mathrm{ap}}^{\mathrm{P}}\right)$ are the same, which we call here "onephospho rate" variant, as opposed to the case when these rates are different, called "two-phospho rates variant". Earlier estimates of the T286 phosphorylation rate at $30^{\circ} \mathrm{C}$ were an order of magnitude lower (Miller and Kennedy 1986; Hanson et al. 1994). Although the "fast" T286 phosphorylation rate is arguably more precise than the "slow" one, we used both in our simulations. Considering that the phosphorylation of exogenous substrates by CaMKII is reduced by $\sim 50 \%$ upon autophosphorylation at T305 (Hashimoto et al. 1987; Lou and Schulman 1989), we set $k_{\mathrm{ap}}^{\mathrm{P}}=0.5 k_{\mathrm{app}}^{\mathrm{P}}$ (we will show below that the exact value is not important). Our simulations of the $\mathrm{Ca}-\mathrm{CaM}-\mathrm{CaMKII}$ equilibrium model showed that for our experimental conditions, $r_{\beta}$ is 3.5-4 times larger than $r_{\alpha}$ (but not bigger than $1)$, for higher and lower $\left[\mathrm{Ca}^{2+}\right]$, respectively. The exact value of $r_{\beta} / r_{\alpha}$ had little influence on the results. The stoichiometry of $\alpha$ and $\beta$ subunits was $3: 1$, as it is in the CNS (Erondu and Kennedy 1985).

For the one-phospho variant, we determined the remaining parameters $\left(r_{\alpha}\right)$ by the parameter search procedure: we ran a series of simulations of the CaMKII phosphorylation model Eqs. 1-3 with different values of the three free parameters $\left(r_{\alpha}\right.$ at three $\left.\left[\mathrm{Ca}^{2+}\right]\right)$ and matched the simulation results to our data from non-phospho T286 $\alpha$ CaMKII Western blots (10 data points at $25^{\circ} \mathrm{C}$ and 9 at $30^{\circ} \mathrm{C}$, points in saturation excluded). In addition, we obtained limiting values $r_{\alpha}$ for two additional $\left[\mathrm{Ca}^{2+}\right]$ at $25^{\circ} \mathrm{C}$ (Fig. 5; Table S4).

Similarly, for the two-phospho variant, we determined the values of the normalized fraction $r_{\alpha}^{(0)}$ at three $\left[\mathrm{Ca}^{2+}\right]$ (and the limiting values at the two additional $\left[\mathrm{Ca}^{2+}\right]$ ), as well as of $k_{\mathrm{ap}}^{\mathrm{P}^{(0)}}\left(\left[\mathrm{Ca}^{2+}\right]\right.$ independent $)$, by a parameter search using the same non-phospho experimental data and Eqs. 14 (Fig. 5; Table S4). We also simulated the experiments presented in Bradshaw et al. (2002) using our value of $k_{\mathrm{ap}}^{\mathrm{P}^{(0)}}$, $r_{\alpha}=r_{\beta}=1$ (these experiments were done at saturating $\mathrm{Ca}^{2+}$ and $\mathrm{CaM}$ ), and the CaMKII autophosphorylation model in order to obtain the phosphorylation rate $k_{\mathrm{aa}}^{\mathrm{P}}$ (and consequently $k_{\mathrm{ap}}^{\mathrm{P}}$, using Eq. 4) for the two-phospho variant. Under these experimental conditions the phosphorylation at T305 does not matter because it was much slower than the phosphorylation at T286. This allowed us to calculate the fraction $r$ at different $\left[\mathrm{Ca}^{2+}\right.$ ] (Table S4) for both fast and slow phosphorylations.

In all cases, we considered a fit to be acceptable if the root mean square error of the simulation in respect to the average values of the experimental data, was smaller or equal to the standard deviation of the data. In cases when the standard deviation of the data was very small the maximum acceptable root mean square errors of the simulations were increased, but did not exceed the error of the best result by more than $50 \%$.

The simulations of the two-phospho rates variant matched the data well (root mean square error of the best simulation results with respect to the experimental data was $0.038)$. Also, we note that the best fit value of the rate $k_{a p}^{\mathrm{P}^{(0)}}$ was much smaller than one, which for the previously determined T286 autophosphorylation rates (Table S5) translates into the active non-phospho subunit T286 phosphorylation rate being significantly higher than the T286 phosphorylated subunit rate. The simulations of the onephospho rate variant did not match the data as well as the two rates variant (root mean square error 0.052 for fast and 0.067 for the slow T286 phosphorylation variant). Furthermore, the slopes of the best-fit line for the errors of the two- and one-phospho variants (calculated as the difference between the predicted values and the experimental data, for all $\mathrm{Ca}^{2+}$ concentrations) were $-0.0057 / \mathrm{min}$ and $0.0327 /$ min, respectively. The difference between them is statistically significant (at 0.94 confidence level, two-tailed $t$ test) arguing that the two fits are different. More importantly, the slope for the one-phospho variant was significantly different from 0 (at 0.90 confidence level, two-tailed $t$ test), while the slope for the two-phospho 

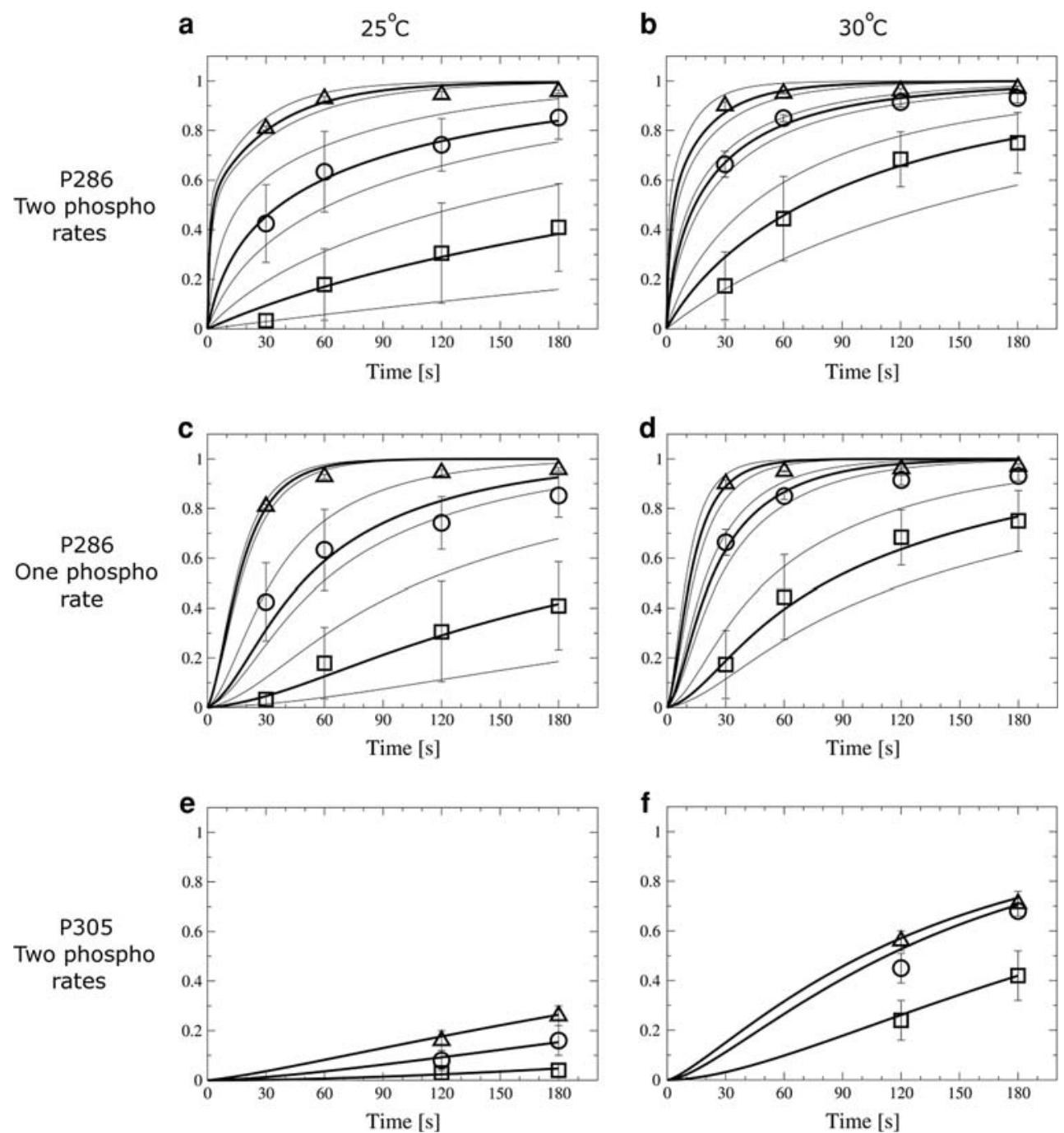

Fig. 5 Simulations of the CaMKII autophosphorylation model. a, b autophosphorylation at T286, experimental data and two-phospho rates variant results. c, d autophosphorylation at T286, experimental data and one-phospho rate, fast phosphorylation variant results. e, f phosphorylation at T305, experimental data and two-phospho rates model results. One-phospho rate model produced virtually indistinguishable data. In all cases the $y$-axis values are normalized so that the total $\alpha \mathrm{CaMKII}$ has a value of 1 . a, $\mathbf{c}, \mathbf{e}$ results at $25^{\circ} \mathrm{C}$, experimentally determined time-courses of phosphorylations at T286 and T305 for

model was not, arguing that the one-phospho variant systematically deviates from the data.

The values of $k_{\mathrm{eff}}^{\mathrm{PP}}$ were then determined from the T305 autophosphorylation data (anti-phospho T286 CaMKII Westerns) for all variants (Table S4). They were consistent with the previously determined rates of CaM dissociation from T286 phosphorylated CaMKII (Meyer et al. 1992). Parameter $k_{\mathrm{eff}}^{\mathrm{PP}}$ combines dissociation of CaM from T286 phosphorylated subunits and the subsequent T305 phosphorylation. It was shown that the rate of $\mathrm{T} 305$ phosphorylation falls well above 1/s (as judged by the initial slope of the T305 phosphorylation initiated by the free $\mathrm{Ca}^{2+}$ concentrations of $1.49,2.14$ and $3.47 \mu \mathrm{M}$ at $25^{\circ} \mathrm{C}$ are represented by open square, open circle and open triangleup, respectively. b, d, f results at $30^{\circ} \mathrm{C}$, the experimental time-courses for the free $\mathrm{Ca}^{2+}$ concentrations of $1.37,1.99$ and $3.26 \mu \mathrm{M}$ are represented by open square, open circle and open triangle, respectively. The simulation results for both one-phospho and two-phospho rate variants of the CaMKII autophosphorylation are shown using thick (best fits) and thin lines (limits). The parameters used for the simulations are given in Table S4

EGTA addition shown on Figure 1 in Lou and Schulman 1989). Considering that this is more than 100 times higher than our values of $k_{\mathrm{eff}}^{\mathrm{PP}}$, we can conclude that the T305 phosphorylation immediately follows the CaM dissociation under our experimental conditions, in other words essentially all subunits that are phosphorylated at $\mathrm{T} 286$ but not at T305 are CaM-bound. Our simulations also showed that if the rate of T286 phosphorylation by T305 phosphorylated subunits $\left(k_{\mathrm{app}}^{\mathrm{P}^{(0)}}\right)$ was changed from $50 \%$ to $20 \%$ or $80 \%$ of the value of $k_{\mathrm{ap}}^{\mathrm{P}^{(0)}}$, the maximum change of CaMKII autophosphorylated at T286 was below 0.02 for $25^{\circ} \mathrm{C}$ and below 0.05 for $30^{\circ} \mathrm{C}$ (total $\alpha$ CaMKII was normalized to 1 ). 
This was expected because under our experimental conditions the T286 phosphorylation occurs at a much faster rate than the T305 phosphorylation. Therefore, the uncertainty of the values of the T305 phosphorylation related rates, $k_{\mathrm{app}}^{\mathrm{P}}$ and $k_{\mathrm{eff}}^{\mathrm{PP}}$, did not affect the values of the T286 phosphorylation related-parameters $k_{\mathrm{aa}}^{\mathrm{P}}, k_{\mathrm{ap}}^{\mathrm{P}}$, and $r_{\alpha}$.

\section{Determination of $\mathrm{Ca}-\mathrm{CaM}-\mathrm{CaMKII}$ binding constants}

We considered three alternatives for the number of CaMbound $\mathrm{Ca}^{2+}$ ions that is sufficient to activate non-phospho CaMKII, namely 2, 3 or 4 ions, and proceeded to clarify this issue by distinguishing between the three corresponding variants: 2-, 3- and 4-activation.

As mentioned above, our numerical simulations indicated that the product of all four affinity increase factors $\left(s_{1} s_{2} s_{3} s_{4}\right)$ falls between 16 and 1,000 , consistent with the reported 40-fold increase of the $\mathrm{Ca}^{2+}$ dissociation rate from $\mathrm{CaM}-\mathrm{CaMKII}$ in the presence of the $\mathrm{CaM}$ binding protein PEP-19 (Putkey et al. 2003; Gaertner et al. 2004b). Also, we required all four of $s_{1}-s_{4}$ to be greater or equal to 1 , meaning that the affinity of CaMKII for CaM does not decrease upon $\mathrm{Ca}^{2+}$ binding to CaM. This is consistent with the models of CaM binding to MLCK, PDE and Phosphorylase b kinase (Burger et al. 1983; Mamar-Bachi and Cox 1987; Cox 1988).

There are two important simplifications that we can make. First, the time scales of the Ca-CaM-CaMKII binding and the T286 autophosphorylation under our experimental conditions were very different. Numerical simulations showed that (using the wide range of values for the affinity increase factors $s_{1}-s_{4}$, see below) the Ca-CaMCaMKII reaction system reached equilibrium with a time constant that is below $0.1 \mathrm{~s}$. On the other hand, the fastest T286 autophosphorylation occurred at a time constant of $10 \mathrm{~s}$, as determined from the initial $10 \mathrm{~s}$ at the highest $\mathrm{Ca}^{2+}$ concentration (Fig. 5). The reason that this time constant was much longer than the T286 phosphorylation rate (Table S5) is that the probability of having two active nonphospho subunits neighboring each other was very low for the actual reactant concentrations. Therefore, the two time scales are well separated and the $\mathrm{Ca}-\mathrm{CaM}-\mathrm{CaMKII}$ reaction system was in partial equilibrium with respect to the T286 autophosphorylation.

Second, the concentration of active non-phospho CaMKII obtained from the $\mathrm{Ca}-\mathrm{CaM}-\mathrm{CaMKII}$ equilibrium model depends on the total non-phospho CaMKII concentration, and it therefore decreases during the course of our experiments, as the total non-phospho CaMKII concentration decreases. Nevertheless, our numerical simulations showed that the fractions $r_{\alpha}$ and $r_{\beta}$ (ratios of active non-phospho and total non-phospho CaMKII concentrations) changed very little during the time course of our experiments, so they could be assumed to be timeindependent and used for the parameter search in the $\mathrm{Ca}-$ CaM-CaMKII equilibrium model.

In order to determine which variant combinations are compatible with our data, we performed exhaustive parameter searches for the values of free parameters $s_{1}-s_{4}$. The simulation results were matched against the values of $r_{\alpha}$ obtained above (Table S4). We investigated 4-, 3-and 2-activation variants in combination with one and twophospho variants, for the two values of $K_{\alpha 4}$, and for the two T286 phosphorylation rates specified above, making 24 different combinations in total. It turned out that only the product of $s_{1}$ and $s_{2}$ mattered, and not their individual values, so the search was simplified by setting $s_{1}=s_{2}$. We also searched for the free parameters that fit the "relaxed" conditions where the minimum and the maximum of the acceptable values was lowered and increased, respectively, by $20 \%$.

The results (Table 1) showed that the combination of the 3 -activation, two-phospho rates, fast phosphorylation, and $1 / K_{\alpha 4}=20 \mathrm{nM}$ variants provided the best fit (constraints

Table 1 Validity of different variants of the six-subunit CaMKII activation and autophosphorylation model

\begin{tabular}{|c|c|c|c|c|c|c|c|c|}
\hline \multirow{4}{*}{$\begin{array}{l}\text { T286 phosphorylation } \\
1 / K_{\alpha 4}(\mathrm{nM}) \\
\mathrm{T}\left[{ }^{\circ} \mathrm{C}\right]\end{array}$} & \multicolumn{4}{|c|}{ Two-phospho } & \multicolumn{4}{|c|}{ One-phospho } \\
\hline & \multicolumn{2}{|l|}{ Fast } & \multicolumn{2}{|l|}{ Slow } & \multicolumn{2}{|l|}{ Fast } & \multicolumn{2}{|l|}{ Slow } \\
\hline & 20 & 60 & 20 & 60 & 20 & 60 & 20 & 60 \\
\hline & $25 / 30$ & $25 / 30$ & $25 / 30$ & $25 / 30$ & $25 / 30$ & $25 / 30$ & $25 / 30$ & $25 / 30$ \\
\hline 4-activation & $-1-$ & $-1-$ & $-1-$ & $-1-$ & $\pm /-$ & $-1-$ & $-1-$ & $-1-$ \\
\hline 3 -activation & $+/+$ & $\pm /+$ & $\pm / \pm$ & $-1-$ & $\pm / \pm$ & $\pm / \pm$ & $+/ \pm$ & $-1-$ \\
\hline 2-activation & $\pm /+$ & $\pm /+$ & $\pm /+$ & $-1+$ & $-1-$ & $-1-$ & $+/ \pm$ & $+/ \pm$ \\
\hline
\end{tabular}

Results obtained from numerical simulations of the 4-, 3-, and 2-activation variants of the Ca-CaM-CaMKII equilibrium model for two values of $1 / K_{\alpha 4}$, and for two values of the T286 phosphorylation rate were matched against the values of $r_{\alpha}$ obtained for one- and two-phospho rates variants of the six-subunit CaMKII autophosphorylation model for both 25 and $30^{\circ} \mathrm{C}$. " + " indicates that it was possible to find the affinity increase factors $s_{1}-s_{4}$ so that the variant fully satisfies the constraints, " \pm " that it was possible to find the affinity increase factors $s_{1}-s_{4}$ so that the variant satisfies the relaxed, but not full, constraints, and "-" that it was not possible to find the affinity increase factors $s_{1}-s_{4}$ so that the variants satisfies the constraints 
were fully satisfied at both 25 and $30^{\circ} \mathrm{C}$ ). The 4 -activation was not consistent with the experimental data (that is, there was no combination of parameters $s_{1}-s_{4}$ that fit the data even when using the relaxed conditions for both temperatures) neither for the one-nor for two-phospho rate variants. Both the 3- and 2-activation variants were consistent with the relaxed conditions when used in different combination with other variants. As expected, the simulation results using the lower affinity of CaMKII for 4-bound CaM, or the slower T286 phosphorylation showed a slight tendency towards the activation variants that need more $\mathrm{Ca}^{2+}$ ions bound.

We also obtained values of the affinity increase factors $s_{1}-s_{4}$, however they could not be determined uniquely (Table 2; Fig. S1). The choice of the activation and phospho rate variants had decisive influence on the validity of the fits and the values of the affinity factors, while $K_{\alpha 4}$, and the T286 phosphorylation rate had moderate influence.

We stress that this parameter search was not an arbitrary data fit. Instead, it was strongly constrained by the $\mathrm{Ca}-$ CaM-CaMKII binding model itself. Furthermore, the parameter search was exhaustive, allowing us to correctly identify variants that are consistent with our data.

The complete dynamic model of CaMKII activation and autophosphorylation

The complete dynamic model of CaMKII activation and autophosphorylation was obtained by combining the equations forming the (non-equilibrium) $\mathrm{Ca}-\mathrm{CaM}-\mathrm{CaMKII}$ binding model (Fig. 3) with the CaMKII autophosphorylation model (Eqs. 1-3). The equations remained unchanged, except that the concentrations of active nonphospho $\alpha$ and $\beta$ CaMKII (or equivalently fractions $\mathrm{r}_{\alpha}$ and $r_{\beta}$ ) in Eqs. 1-2 were treated dynamically, and the terms defining the T286 phosphorylation of active subunits were added to the equations of the $\mathrm{Ca}-\mathrm{CaM}-\mathrm{CaMKII}$ binding. Therefore, the simplifications that were made earlier $(\mathrm{Ca}-$ CaM-CaMKII binding in partial equilibrium and the timeindependence of $r_{\alpha}$ and $r_{\beta}$ ) were not needed. The complete model consists of 56 first-order non-linear differential equations, and had the same number of variables (42 from the T286 autophosphorylation model and 15 from the $\mathrm{Ca}-$ CaM-CaMKII binding model, minus one imposing $\mathrm{CaM}$ conservation).

Numerical simulations of this model used the same values binding constants and phosphorylation rates that were used in the simulations of the $\mathrm{Ca}-\mathrm{CaM}-\mathrm{CaMKII}$ binding and of the CaMKII autophosphorylation models. The resulting time-courses for $\alpha$ CaMKII autophosphorylation at T286 and T305 matched our experimental data, as well as the results of the CaMKII autophosphorylation model (Fig. 5), thus confirming the validity of the above two simplifications for our experimental conditions.

The complete dynamic CaMKII model is not restricted to certain experimental conditions. It can simulate the CaMKII autophosphorylation under any reagent concentrations. We used the two-phospho, fast phosphorylation variant combination and simulated the T286 phosphorylation for different $[\mathrm{CaM}]$ at the saturating $\mathrm{Ca}^{2+}$, for the reactant concentrations employed in (Gaertner et al. 2004a, Figure 6A). The results were fitted to the Hill equations to obtain Hill coefficient of 2.0 (Fig. S2), in agreement with the experimental data.

Sensitivity of the simulations to the number of subunits

In order to investigate how the number of subunits in the CaMKII holoenzyme influences the T286 phosphorylation

Table 2 Representative values of the affinity increase factors $s_{1}-s_{4}$ for the six-subunit model

\begin{tabular}{|c|c|c|c|c|c|c|c|c|c|c|c|c|}
\hline \multirow{3}{*}{$\mathrm{T}\left[{ }^{\circ} \mathrm{C}\right]$} & \multicolumn{6}{|c|}{ Two-phospho } & \multicolumn{6}{|c|}{ One-phospho } \\
\hline & \multicolumn{3}{|l|}{25} & \multicolumn{3}{|l|}{30} & \multicolumn{3}{|l|}{25} & \multicolumn{3}{|l|}{30} \\
\hline & $s_{1}$ & $s_{3}$ & $s_{4}$ & $s_{1}$ & $s_{3}$ & $s_{4}$ & $s_{1}$ & $s_{3}$ & $s_{4}$ & $s_{1}$ & $s_{3}$ & $s_{4}$ \\
\hline 3 -act, Fast, 20 & 1 & 64 & 4 & 1 & 45.2 & 4 & 2.4 & 1.4 & 2 & 4.4 & 1 & 4 \\
\hline 3 -act, Fast, 60 & 1 & 64 & 1 & 1 & 64 & 1 & 2.8 & 2 & 1 & 2.8 & 2 & 1 \\
\hline 3-act, Slow, 20 & 2.8 & 128 & 1 & 2.8 & 128 & 1 & 3.2 & 11.3 & 1 & 5.3 & 11.3 & 1 \\
\hline \multicolumn{13}{|l|}{ 3-act, Slow, 60} \\
\hline 2-act, Fast, 20 & 1 & 5.6 & 40.2 & 1 & 6 & 32 & & & & & & \\
\hline 2-act, Fast, 60 & 1 & 64 & 2 & 1 & 64 & 2 & & & & & & \\
\hline 2-act, Slow, 20 & 1 & 64 & 1 & 1.7 & 64 & 1 & 2.5 & 16 & 8 & 1.4 & 8 & 8 \\
\hline 2-act, Slow, 60 & & & & & & & 1.1 & 32 & 1 & 1.6 & 32 & 1 \\
\hline
\end{tabular}

Shown are representative examples of values of $s_{1}-s_{4}$ for all different combinations of the variants that are consistent with our experimental data. In each case, the values shown in this table lay in the middle of the range of values that are consistent with the experimental data. Whenever possible the values for 25 and $30^{\circ} \mathrm{C}$ were chosen to be similar. Left column defines variant combinations: 3- or 4-activation, fast of slow T286 phosphorylation, and two different values of $1 / K_{\alpha 4}(20$ or $60 \mathrm{nM})$ 
rate, we developed two CaMKII autophosphorylation models for seven subunits forming a ring and for subunits functionally organized in pairs. Both models were developed and simulated in the same manner as the 6-subunit model discussed previously.

The results for the 6- and 7-subunit-models were virtually indistinguishable: the relative difference between them did not exceed $1 \%$.

As expected from the fact that the 6-subunit model allows more possibilities of having two neighboring active subunits than the 2-subunit model, in our simulations of the 2-subunit model the phosphorylation of T286 proceeded slower and consequently the fractions $r_{\alpha}$ and $r_{\alpha}^{(0)}$ were higher (Table S6) . Nevertheless, the simulations of the 2subunit model matched our experimental data well (root mean square error of the best simulation results were 0.039 for the two-phospho variant and 0.046 for the one-phospho). Again, the 4-activation variant did not fit our data at all, while the 3- and 2- activation variants did. In all combinations involving the one-phospho variant the values of parameters $s_{1}-s_{4}$ at 25 and $30^{\circ} \mathrm{C}$ were very different, or the values of the affinity increase factors $s_{3}$ and $s_{4}$ were comparable or smaller than $s_{1}$ and $s_{2}$, arguing against the validity of this variant. In all other cases these values were concentrated in regions where the values of $s_{3}$ and $s_{4}$ were much bigger than $s_{1}$ and $s_{2}$ (Tables S7, S8).

\section{Discussion}

In this study, we combined biochemical experiments with computer modeling of biochemical reactions to achieve two goals: to elucidate some of the currently unresolved aspects of CaMKII activation and autophosphorylation that might be relevant for its physiological function and to provide a model that incorporates in detail the mechanism of CaMKII activation and T286 autophosphorylation and is based on experimentally determined binding constants and phosphorylation rates.

In order to achieve these goals, we developed a detailed state model of CaMKII activation and autophosphorylation. The states of this model, and the transitions between them, are based on current knowledge and comprise a detailed representation of the CaMKII autophosphorylation and $\mathrm{Ca}-\mathrm{CaM}-\mathrm{CaMKII}$ binding. The model is valid at physiologically relevant conditions in which the concentrations of $\mathrm{Ca}^{2+}$ and $\mathrm{CaM}$ are not saturating, and it takes into account all essential features of CaMKII activation and autophosphorylation known up to now, namely the holoenzyme structure of CaMKII, the two CaMKII subunit isoforms $(\alpha$ and $\beta)$, their stoichiometry in the CNS, the binding of $\mathrm{Ca}$ to $\mathrm{CaM}$ and of $\mathrm{Ca}$-bound $\mathrm{CaM}$ to both CaMKII isoforms, as well as the intersubunit intraholoenzyme nature of T286 phosphorylation and the intrasubunit nature of T305 phosphorylation. The model distinguishes not only between holoenzymes with different numbers of phosphorylated subunits, but also between different arrangements of phosphorylated subunits within a holoenzyme. Whenever available, the input parameters (binding constants and phosphorylation rates) were taken from the literature, or obtained by numerically simulating published experimental data. However, not all of them could be determined in this way.

We defined several variants of the model, related to the currently unresolved aspects of CaMKII activation and phosphorylation. While in principle active non-phospho and T286 phosphorylated subunits can phosphorylate a neighboring active non-phospho subunit at T286 at different rates (two-phospho rates variant), these rates are assumed to be the same in the current literature (onephospho rate variant). Furthermore, we considered three alternatives for the number of $\mathrm{Ca}^{2+}$ ions that is sufficient to activate CaM-CaMKII (2, 3 or 4 ions), and defined three corresponding variants: 2-, 3- and 4-activation. In addition, we considered two different values for the affinity of $\alpha \mathrm{CaMKII}$ for 4-bound CaM, and two different values for the T286 phosphorylation rate that are currently available in the literature.

The experimental time-courses of $\alpha$ CaMKII autophosphorylations at T286 and T305 for different (nonsaturating) $\mathrm{Ca}^{2+}$ concentrations and temperatures were obtained using quantitative Western blots. Using all variants combinations of model, we preformed exhaustive parameter searches in order to find the values of the previously undetermined input parameters that were consistent with our experimental data. A simplification of our model applicable to our experimental conditions rendered this search manageable.

We found that the 4-activation variant was not consistent with our data, since there was no combination of the affinity increase factors $s_{1}-s_{4}$ that fitted our experimental data (Table 1). Therefore, our data strongly suggests that 3bound CaM is sufficient for the activation of CaMKII but it does not exclude the possibility that CaMKII can be activated by 2-bound CaM. In the case of $\mathrm{CaM}$ binding to MLCK whose CaM binding domain shares homology with the CaM binding domain of CaMKII, the binding of the third $\mathrm{Ca}^{2+}$ is necessary for the activation (Mamar-Bachi and Cox 1987), However, it was recently shown that a modified form of CaM containing two inactivated $\mathrm{Ca}^{2+}$ binding sites can still partially activate CaMKII (Shifman et al. 2006).

The combination of the following lines of evidence argue against the one-phospho variant. The affinity increase factors $s_{3}$ and $s_{4}$ are expected to be significantly higher than $s_{1}$ and $s_{2}$, based on the reported increase of 
CaM affinity for the third and the fourth $\mathrm{Ca}^{2+}$ ion upon MLCK, PDE, and phosphorylase $b$ kinase binding is much higher than that for the binding of the first two $\mathrm{Ca}^{2+}$ ions. Also, it is unlikely that the values of $s_{1}-s_{4}$ at $25^{\circ} \mathrm{C}$ and $30^{\circ} \mathrm{C}$ are much different. Furthermore, it is likely that the fast T286 phosphorylation rate is more precise. Finally, the two-phospho rate variant matched the experimental data significantly better than the one-phospho rate variant. All this taken together suggests that the T286 phosphorylation rate of an active non-phospho (CaM-bound, non-phosphorylated) subunit by an active non-phospho subunit is considerably higher than by a T286 phosphorylated (CaMbound and non-phosphorylated at T305) subunit.

A positive cooperativity between T286 phosphorylation and $\mathrm{CaM}$ concentration was reported in (Gaertner et al. 2004a). We simulated the autophosphorylation assay employed in that publication using the two-phospho variant and obtained the same cooperativity (Fig. S2), arguing that the two-phospho variant is consistent with the current experimental data.

In order to investigate the significance of CaMKII holoenzyme structure on CaMKII autophosphorylation at T286, we developed models in which the subunits are organized so that they can phosphorylate each other within a six-subunit ring (based on the dodecamer structure of CaMKII holoenzyme (Kolodziej et al. 2000; Gaertner et al. 2004a)), seven-subunit ring (based on the 14-subunit structure (Hoelz et al. 2003)), and a two-subunit pair. The six and seven-subunit models gave virtually identical results, and while the values of some parameters were different for the two-subunit model, the conclusions regarding the validity of different variants remained the same. Recently, a CaM-binding model was proposed having cooperativity between pairs of CaMKII subunits belonging to the same holoenzyme but residing on opposite subunit rings (Rosenberg et al. 2005). This model would imply that the distribution of bound CaM's is somewhat skewed against binding of neighboring CaMKII subunits. Regarding our simulations, this might result in a slower rate of the T286 phosphorylation for low T286 phosphorylation levels, arguably affecting the one-phospho variant more than the two-phospho variant (Fig. 5).

We stress that our parameter searches were not data fits with arbitrary functions. Instead, they were strongly constrained by the detailed reaction schemes of CaMKII activation and T286 autophosphorylation. Furthermore, these parameter searches were exhaustive, thus making sure that our conclusions concerning the validity of different variants in respect to our experimental data were properly supported by the data.

The prominent increase of CaM affinity for the third $\mathrm{Ca}^{2+}$ ion (the first low affinity site) upon CaMKII binding, obtained for the combination of two-phospho and 2- or 3-activation variants, increases affinities of the low affinity sites, and strongly suggests that CaMKII is efficiently activated at $\mathrm{Ca}^{2+}$ concentrations that are lower than what could be expected based on the CaM affinity for $\mathrm{Ca}^{2+}$. This finding might help explain how CaMKII gets activated at neuronal synapses in vivo, considering that the maximal $\mathrm{Ca}^{2+}$ concentration reached is expected to be in the low $\mu \mathrm{M}$ range, similar to the values of the dissociation constant for the binding of the last $\mathrm{Ca}^{2+}$ ion needed to activate $\mathrm{CaM}-$ CaMKII that we obtained (Table 2). Furthermore, findings that CaMKII can translocate to synapses upon stimulation (Shen and Meyer 1999), and that the binding to synaptic NMDA receptors activates bound CaMKII subunits and requires activation by $\mathrm{CaM}$ binding of only one additional subunit on a CaMKII ring (and not two neighboring subunits as is otherwise the case) (Bayer et al. 2001), suggest that the probability of (NMDA receptor-bound) CaMKII autophosphorylation becomes high upon the stimulationinduced opening of NMDA receptors. Additionally, the lower rate of T286 phosphorylation by T286 phosphorylated subunits might slow down further T286 phosphorylation and may avoid the phosphorylation of the whole holoenzyme. Therefore, not only the $\mathrm{Ca}^{2+}$ concentration, but also the exact form of the $\mathrm{Ca}^{2+}$ signal (for example, $\mathrm{Ca}^{2+}$ influx through NMDA receptors/channels is different from the influx through the voltage gated $\mathrm{Ca}^{2+}$ channels at the postsynaptic sides of excitatory neurons in the CNS) may determine the activity of CaMKII in vivo.

Considering the complexity of the signaling pathways at neuronal synapses, computer simulations may be required to acquire a more detailed, quantitative understanding of these processes. The model developed here, including the values of the previously undetermined biochemical constants, could be used as an important component in simulations of networks of interconnected signaling pathways that are present at neuronal synapses, leading to a better understanding of biochemical processes underlying synaptic plasticity.

We hope that this model can be used by the growing community of scientists that are ready to investigate more complicated signaling pathways occurring in a living cell. In combination with further biochemical experiments, our model could in future be used or extended to further constrain some of the binding constants or phosphorylation rates involved in the CaMKII activation and autophosphorylation, or to provide the detailed reaction scheme for the CaM dissociation from the CaMKII autophosphorylated at $\mathrm{T} 286$.

Acknowledgments The work was supported by a fellowship to V. L. from the Caltech computational Biology Initiative funded by a Burroughs Wellcome Fund Interfaces Award and by grant no. NS44306 to M.B.K. from the US Public Health Service National Institutes of Health. We wish to thank Alan J. Rosenstein for technical 
assistance. V. L. would like to acknowledge the generous support of W. Baumeister.

Open Access This article is distributed under the terms of the Creative Commons Attribution Noncommercial License which permits any noncommercial use, distribution, and reproduction in any medium, provided the original author(s) and source are credited.

\section{References}

Bayer K, De Koninck P, Leonard A, Hell J, Schulman H (2001) Interaction with the NMDA receptor locks CaMKII in an active conformation. Nature 411:801-805

Bennett MK, Erondu NE, Kennedy MB (1983) Purification and characterization of a calmodulin-dependent protein kinase that is highly concentrated in brain. J Biol Chem 258:12735-12744

Bollmann J, Sakmann B, Borst J (2000) Calcium sensitivity of glutamate release in a calyx-type terminal. Science 289:953-957

Bradshaw JM, Hudmon A, Schulman H (2002) Chemical quenched flow kinetic studies indicate an intraholoenzyme autophosphorylation mechanism for $\mathrm{Ca}^{2+} /$ calmodulin-dependent protein kinase II. J Biol Chem 277:20991-20998

Bradshaw JM, Kubota Y, Meyer T, Schulman H (2003) An ultrasensitive $\mathrm{Ca}^{2+}$ /calmodulin-dependent protein kinase II-protein phosphatase 1 switch facilitates specificity in postsynaptic calcium signaling. Proc Natl Acad Sci USA 100:10512-10517

Brocke L, Chiang LW, Wagner PD, Schulman H (1999) Functional implications of the subunit composition of neuronal CaM kinase II. J Biol Chem 274:22713-22722

Burger D, Stein EA, Cox JA (1983) Free energy coupling in the interactions between $\mathrm{Ca}^{2+}$, calmodulin, and phosphorylase kinase. J Biol Chem 258:14733-14739

Cox JA (1988) Interactive properties of calmodulin. Biochem J 249:621-629

Dosemeci A, Albers RW (1996) A mechanism for synaptic frequency detection through autophosphorylation of cam kinase ii. Biophys J 70:2493-2501

Dupont G, Houart G, De Koninck P (2003) Sensitivity of cam kinase ii to the frequency of ca2+ oscillations: a simple model. Cell Calcium 34:485-497

Erondu NE, Kennedy MB (1985) Regional distribution of type II $\mathrm{Ca}^{2+} /$ calmodulin-dependent protein kinase in rat brain. $\mathrm{J}$ Neurosci 5: 3270-3277

Gaertner TR, Kolodziej SJ, Wang D, Kobayashi R, Koomen JM, Stoops JK, Waxham MN (2004a) Comparative analyses of the three-dimensional structures and enzymatic properties of alpha, beta, gamma and delta isoforms of $\mathrm{Ca}^{2+}$-calmodulin-dependent protein kinase II. J Biol Chem 279:12484-12494

Gaertner TR, Putkey JA, Waxham MN (2004b) RC3/Neurogranin and $\mathrm{Ca}^{2+} /$ calmodulin-dependent protein kinase II produce opposing effects on the affinity of calmodulin for calcium. J Biol Chem 279:39374-39382

Gregori L, Gillevet PM, Doan P, Chau V (1985) Mechanism of enzyme regulation by calmodulin and $\mathrm{Ca}^{2+}$. Curr Top Cell Regul 27:447-454

Hanson PI, Meyer T, Stryer L, Schulman H (1994) Dual role of calmodulin in autophosphorylation of multifunctional cam kinase may underlie decoding of calcium signals. Neuron 12:943-956

Hashimoto Y, Schworer CM, Colbran RJ, Soderling TR (1987) Autophosphorylation of $\mathrm{Ca}^{2+} /$ calmodulin-dependent protein kinase II. effects on total and $\mathrm{Ca}^{2+}$-independent activities and kinetic parameters. J Biol Chem 262:8051-8055
Hayashi Y, Shi SH, Esteban JA, Piccini A, Poncer JC, Malinow R (2000) Driving AMPA receptors into synapses by LTP and CaMKII: requirement for GluR1 and PDZ domain interaction. Science 287:2262-2267

Hoelz A, Nairn AC, Kuriyan J (2003) Crystal structure of a tetradecameric assembly of the association domain of $\mathrm{Ca}^{2+}$ / calmodulin-dependent kinase II. Mol Cell 11:1241-1251

Holmes WR (2000) Models of calmodulin trapping and CaM kinase II activation in a dendritic spine. J Comput Neurosci 8:6585

Klee C (1988) Interaction of calmodulin with $\mathrm{ca}^{2+}$ and target proteins. In: Cohen $\mathrm{P}$, Klee $\mathrm{C}$ (eds) Calnodulin. Elsevier, Amsterdam, pp 35-56

Kolodziej SJ, Hudmon A, Waxham MN, Stoops JK (2000) Threedimensional reconstructions of calcium/calmodulin-dependent $(\mathrm{CaM})$ kinase iialpha and truncated CaM kinase IIalpha reveal a unique organization for its structural core and functional domains. J Biol Chem 275:14354-14359

Kubota Y, Bower JM (2001) Transient versus asymptotic dynamics of CaM kinase II: possible roles of phosphatase. J Comput Neurosci 11:263-279

Lou LL, Schulman H (1989) Distinct autophosphorylation sites sequentially produce autonomy and inhibition of the multifunctional $\mathrm{Ca}^{2+} /$ calmodulin-dependent protein kinase. J Neurosci 9:2020-2032

Lou LL, Lloyd SJ, Schulman H (1986) Activation of the multifunctional $\mathrm{Ca}^{2+} /$ calmodulin-dependent protein kinase by autophosphorylation: ATP modulates production of an autonomous enzyme. Proc Natl Acad Sci USA 83:9497-9501

Lukas T, Mirzoeva S, Watterson D (1998) Calmodulin-regulated protein kinases. In: Eldik LV, Watterson D (eds) Calmodulin and Signal Transduction. Academic Press, London, pp 65-168

Mamar-Bachi A, Cox JA (1987) Quantitative analysis of the free energy coupling in the system calmodulin, calcium, smooth muscle myosin light chain kinase. Cell Calcium 8:473-482

Martell AE, Smith RM (1982) Critical stability constants, vol 1: amino acids. Plenum Press, NY, p 269

Meyer T, Hanson PI, Stryer L, Schulman H (1992) Calmodulin trapping by calcium-calmodulin-dependent protein kinase. Science 256:1199-1202

Miller SG, Kennedy MB (1985) Distinct forebrain and cerebellar isozymes of type II $\mathrm{Ca}^{2+}$ /calmodulin-dependent protein kinase associate differently with the postsynaptic density fraction. J Biol Chem 260:9039-9046

Miller SG, Kennedy MB (1986) Regulation of brain type ii $\mathrm{Ca}^{2+} /$ calmodulin-dependent protein kinase by autophosphorylation: a $\mathrm{Ca}^{2+}$-triggered molecular switch. Cell 44:861-870

Miller SG, Patton BL, Kennedy MB (1988) Sequences of autophosphorylation sites in neuronal type ii cam kinase that control $\mathrm{Ca}^{2+}$-independent activity. Neuron 1:593-604

Miller P, Zhabotinsky AM, Lisman JE, Wang XJ (2005) The stability of a stochastic camkii switch: dependence on the number of enzyme molecules and protein turnover. PLoS Biol 3:e107

Okamoto H, Ichikawa K (2000) Switching characteristics of a model for biochemical-reaction networks describing autophosphorylation versus dephosphorylation of $\mathrm{Ca}^{2+} /$ calmodulin-dependent protein kinase II. Biol Cybern 82:35-47

Patton BL, Miller SG, Kennedy MB (1990) Activation of type ii calcium/calmodulin-dependent protein kinase by $\mathrm{Ca}^{2+} /$ calmodulin is inhibited by autophosphorylation of threonine within the calmodulin-binding domain. J Biol Chem 265:11204-11212

Patton BL, Molloy SS, Kennedy MB (1993) Autophosphorylation of type ii cam kinase in hippocampal neurons: localization of phospho- and dephosphokinase with complementary phosphorylation site-specific antibodies. Mol Biol Cell 4:159-172 
Putkey JA, Kleerekoper Q, Gaertner TR, Waxham MN (2003) A new role for IQ motif proteins in regulating calmodulin function. J Biol Chem 278:49667-49670

Rich RC, Schulman H (1998) Substrate-directed function of calmodulin in autophosphorylation of $\mathrm{Ca}^{2+} /$ calmodulin-dependent protein kinase II. J Biol Chem 273:28424-28429

Rosenberg O, Deindl S, Sung R, Nairn A, Kuriyan J (2005) Structure of the autoinhibited kinase domain of CaMKII and SAXS analysis of the holoenzyme. Cell 123:849-860

Sabatini B, Oertner T, Svoboda K (2002) The life cycle of $\mathrm{Ca}(2+)$ ions in dendritic spines. Neuron 33:439-452

Schneggenburger R, Neher E (2000) Intracellular calcium dependence of transmitter release rates at a fast central synapse. Nature 406:889-893

Shen K, Meyer T (1999) Dynamic control of CaMKII translocation and localization in hippocampal neurons by NMDA receptor stimulation. Science 284:162-166

Shifman J, Choi M, Mihalas S, Mayo S, Kennedy M (2006) Ca2+/ calmodulin-dependent protein kinase II (CaMKII) is activated by calmodulin with two bound calciums. Proc Natl Acad Sci USA 103:13968-13973
Silva AJ, Stevens CF, Tonegawa S, Wang Y (1992) Deficient hippocampal long-term potentiation in alpha-calcium-calmodulin kinase II mutant mice. Science 257:201-206

Singla SI, Hudmon A, Goldberg JM, Smith JL, Schulman H (2001) Molecular characterization of calmodulin trapping by calcium/ calmodulin-dependent protein kinase ii. J Biol Chem 276:29353-29360

Smith PD, Liesegang GW, Berger RL, Czerlinski G, Podolsky RJ (1984) A stopped-flow investigation of calcium ion binding by ethylene glycol bis(beta-aminoethyl ether)-N,N'-tetraacetic acid. Anal Biochem 143:188-195

Stevens CF, Tonegawa S, Wang Y (1994) The role of calciumcalmodulin kinase II in three forms of synaptic plasticity. Curr Biol 4:687-693

Wang JH, Sharma RK, Huang CY, Chau V, Chock PB (1980) On the mechanism of activation of cyclic mucleotide phosphodiesterase by calmodulin. Ann N Y Acad Sci 356:190-204

Zhabotinsky AM (2000) Bistability in the $\mathrm{Ca}^{2+} /$ calmodulin-dependent protein kinase-phosphatase system. Biophys J 79:22112221 